Conference presentation: SIR Congress 2014

\title{
Joint involvement in patients affected by systemic lupus erythematosus: application of the swollen to tender joint count ratio
}

\author{
E. Cipriano, F. Ceccarelli, L. Massaro, F.R. Spinelli, C. Alessandri, \\ C. Perricone, G. Valesini, F. Conti \\ Lupus Clinic, Rheumatology, Dipartimento di Medicina e Specialità Mediche, \\ La Sapienza University of Rome, Italy
}

\section{SUMMARY}

Joint involvement is a common manifestation in systemic lupus erythematosus (SLE). According to the SLE disease activity index 2000 (SLEDAI-2K), joint involvement is present in case of $\geq 2$ joints with pain and signs of inflammation. However this definition could fail to catch all the various features of joint involvement. Alternatively the Swollen to Tender joint Ratio (STR) could be used. This new index, which was originally proposed for rheumatoid arthritis (RA) patients, is based on the count of 28 swollen and tender joints. Our study is, therefore, aimed to assess joint involvement in a SLE cohort using the STR.

SLE patients with joint symptoms ( $\geq 1$ tender joint) were enrolled over a period of one month. Disease activity was assessed by SLEDAI-2K. We performed the swollen and tender joint count (0-28) and calculated the STR. Depending on the STR, SLE patients were grouped into three categories of disease activity: low $(\mathrm{STR}<0.5)$, moderate $(\leq 0.5 \mathrm{STR} \leq 1.0)$, high $(\mathrm{STR}>1.0)$. We also calculated the disease activity score based on a 28 -joint count and the erythrocyte sedimentation rate (DAS28-ESR).

We enrolled 100 SLE patients [F/M 95/5, mean \pm standard deviation (SD) age 46.3 \pm 10.6 years, mean \pm SD disease duration 147.1 \pm 103.8 months]. The median of tender and swollen joints was 4 (IQR 7) and 1 (IQR 2.5), respectively. The median STR value was 0.03 (IQR 0.6 ). According to the STR, disease activity was low in 70 patients, moderate in 23 and high in 7 . A significant correlation was identified between STR values and DAS28 $(\mathrm{r}=0.33, \mathrm{p}=0.001)$.

The present study suggests a correlation between STR and DAS28, allowing an easier and faster assessment of joint involvement with the former index.

Key words: Systemic lupus erythematosus; joint involvement; swollen to tender joint ratio.

Reumatismo, 2015; 67 (2): 62-67

\section{INTRODUCTION}

oint involvement is a common manifes$\mathcal{J}$ tation in patients affected by systemic lupus erythematosus (SLE) $(1,2)$.

The SLE disease activity index 2000 (SLEDAI-2K) is the most frequently used index in the clinical practice for SLE patients (3).

However, the SLEDAI-2K indicates the presence of joint involvement only in case of $\geq 2$ joints with pain and signs of inflammation, such as tenderness, swelling or effusion (3).

This stringent definition may, therefore, fail to identify all the different forms of joint involvement.

Kristensen et al. have recently proposed a new index for rheumatoid arthritis (RA) resulting from a ratio of swollen to tender joints based on a 28 joint count named swollen to tender joint count ratio (STR) (4).

This index seems reliable and easy to calculate in the clinical routine, can identify the different degrees of disease activity and does not require the erythrocyte sedimentation rate (ESR) and the patient's assessment, which could be influenced by other factors in SLE patients. 
Therefore in this study we aimed to assess joint involvement in a large SLE cohort using the STR clinical marker.

\section{MATERIALS AND METHODS}

During one month, we enrolled 100 consecutive SLE patients with active joint complaints ( $\geq 1$ tender joint). All patients were referred to the Lupus Clinic, Rheumatology Unit, La Sapienza University of Rome.

The study protocol was in compliance with the principle of good clinical practice and the Declaration of Helsinki statements. All patients gave their informed consent to participate in the study. The ethical committee of Sapienza University of Rome approved the study protocol.

The diagnosis of SLE was made on the basis of the 1997 American College of Rheumatology (ACR) revised criteria (5). Clinical and laboratory data, as well as demographics and past medical history with date of diagnosis, comorbidities and previous and concomitant treatments were reported in a standardized electronic form. All patients underwent a complete assessment, including a global health assessment by a visual analogue scale $(\mathrm{GH} ; 0-100$ $\mathrm{mm}$ ).

Peripheral blood samples were collected from all patients to evaluate the autoantibody profile and complement serum levels. Specifically, anti-dsDNA antibodies were assessed by indirect immunoflorescence on Crithidia Luciliae in accordance with the manufacturer's instructions (Orgentec Diagnostika, Mainz, Germany). Serum levels of complement $\mathrm{C} 3$ and $\mathrm{C} 4(\mathrm{mg} / \mathrm{dL})$ were examined by radial immunodiffusion. ESR was determined with standard methods $(\mathrm{mm} / \mathrm{h}$, Westergren). Disease activity was assessed by using the SLEDAI-2K at the time of the visit (3).

\section{Joint assessment}

We initially calculated the STR, which is a ratio based on the count of 28 swollen and tender joints. According to the STR values, SLE patients were grouped into three categories of disease activity: low
(STR $<0.5)$, moderate $(\leq 0.5$ STR $\leq 1.0)$, high (STR >1.0) (4). The disease activity score (28-joint count, four variables, ESRbased; DAS28) was calculated. According to DAS28 values, joint disease activity was defined as low (DAS28 $\leq 3.2$ ), moderate $(<3.2$ DAS28 $\leq 5.1)$ or high $($ DAS28 $>5.1)$ (6). In the end, the remission status coincided with a DAS28 <2.6(7).

\section{Statistical analysis}

The software packages we used were MedCalc 16.0 (MedCalc Software, Mariakerke, Belgium) and Statistical Package for Social Sciences (SPSS 13.0, Chicago, IL, USA). Data were reported as means and standard deviations (SD) or medians

Table I - Historical clinical, laboratory and therapeutical features of the 100 systemic lupus erythematosus patients.

\begin{tabular}{|c|c|}
\hline Patients features & Frequency \\
\hline $\begin{array}{l}\text { Clinical manifestation } \\
\text { Skin involvement } \\
\text { Serositis } \\
\text { Hematological involvement } \\
\text { Renal involvement } \\
\text { Neuropsychiatric involvement }\end{array}$ & $\begin{array}{c}63 \% \\
20 \% \\
51 \% \\
27 \% \\
8 \%\end{array}$ \\
\hline $\begin{array}{l}\text { Laboratory evaluation } \\
\text { Anti-nuclear antibodies } \\
\text { Anti-double stranded DNA } \\
\text { Anti-Sm } \\
\text { Anti-SSA } \\
\text { Anti-SSB } \\
\text { Anti-RNP } \\
\text { Anti-cardiolipin lgG/lgM } \\
\text { Anti- } \beta 2 \text {-glycoprotein } 1 \text { lgG/lgM } \\
\text { Lupus anticoagulant } \\
\text { Low complement levels } \\
\text { (C3 and/or C4) }\end{array}$ & $\begin{array}{c}15 \% \\
36 \% \\
12 \% \\
17 \% \\
22 \% \\
8 \% \\
12 \% \\
50 \%\end{array}$ \\
\hline $\begin{array}{l}\text { Therapy } \\
\text { Glucocorticoids } \\
\text { Hydroxychloroquine } \\
\text { Methotrexate } \\
\text { Azathioprine } \\
\text { Cyclosporine A } \\
\text { Mycophenolate mofetil } \\
\text { Cyclophosphamide } \\
\text { Rituximab } \\
\text { Glucocorticoid dosage mg/week } \\
\text { (mean, range) }\end{array}$ & $\begin{array}{c}100 \% \\
64 \% \\
30 \% \\
28 \% \\
20 \% \\
35 \% \\
3 \% \\
3 \% \\
87,5 \mathrm{mg} \\
(0-175)\end{array}$ \\
\hline
\end{tabular}




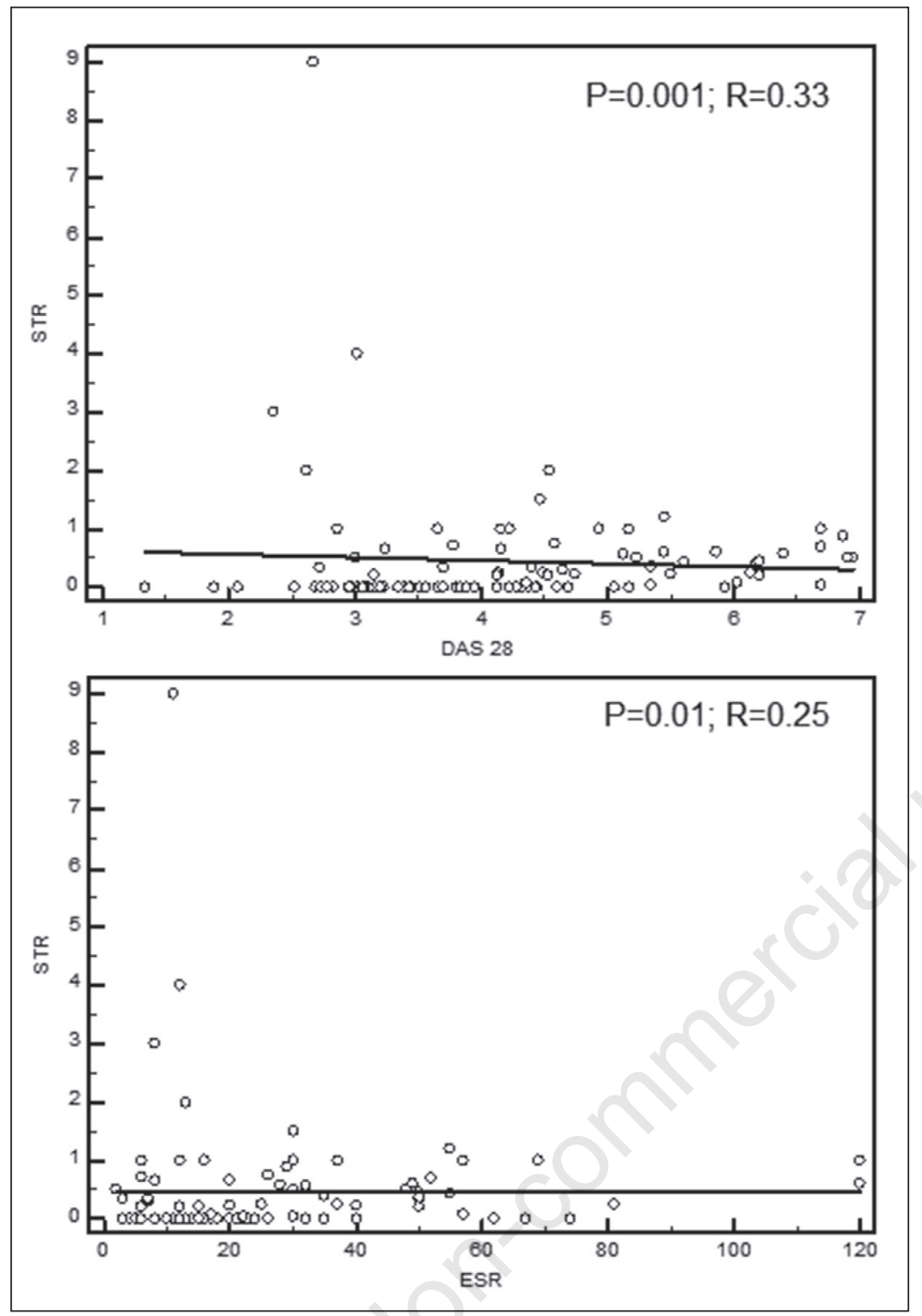

Figure 1 - Correlation between swollen to tender joint count ratio (STR) and disease activity score 28-joint count (DAS28) values and ESR.

with an interquantile range (IQR) depending on the data distribution (tested with the Kolmogorov-Smirnov test). Histograms were used to visualize the distribution of swollen and tender joints, STR and STR categories. Pearson's and Spearman's tests were used to perform correlation analyses when appropriate. Wilcoxon's matched pairs test and paired t-test were performed accordingly. Univariate comparisons between nominal variables were calculated using the chi-square test or Fisher's exact test when appropriate. Two-tailed $\mathrm{p}$ values were reported; $p$ values $\leq 0.05$ were considered statistically significant.

\section{RESULTS}

We enrolled 100 SLE patients (M/F 5/95, mean \pm SD age $46.3 \pm 10.6$ years, mean \pm SD disease duration $147.1 \pm 103.8$ months). Table I reports the main features of the enrolled patients. Firstly, we counted the swollen and tender joints and calculated a median of 1 (IQR 2.5) and 4 (IQR 7), respectively. Subsequently, we calculated the STR values, which gave a median of 0.03 (IQR 0.6).

According to STR values, the disease activity was low in 70 patients, moderate in 23 and high in 7. We calculated the DAS28, which gave a median of 4.1 (IQR 1.96).

We also observed a positive correlation between STR and DAS28 values $(\mathrm{p}=0.001$, $\mathrm{r}=0.33$; Figure 1) and between STR and $\operatorname{ESR}(\mathrm{p}=0.01 ; \mathrm{r}=0.25)$. We grouped SLE patients according to the disease activity identified by STR values [low (STR $<0.5)$, moderate $(\leq 0.5$ STR $\leq 1.0)$, high (STR $>1.0)]$ and performed a comparison among the features of the three groups. Table II reports only the comparisons with statistically significant results.

We evaluated 34 patients with joint involvement identified by SLEDAI-2K.

STR was low in $29.4 \%$ of them, moderate in $50.0 \%$ and severe in $20.6 \%$ (Figure 2A).

The remaining 66 patients without joint involvement defined by SLEDAI-2K had a low STR in $91 \%$ of cases and a moderate STR in $9 \%$ of cases (Figure 2B).

\section{DISCUSSION AND CONCLUSIONS}

In this study, the STR clinical marker was used for the first time to evaluate joint involvement in SLE patients. Our results demonstrate a significant correlation between the STR and DAS28, suggesting the possibility to use it as an easier and faster index. Moreover, STR seems to be more sensitive than SLEDAI-2K in capturing joint involvement.

SLE is a systemic autoimmune disease characterized by a multi-factorial etiology, a broad autoantibody profile and het- 
Table II - Demographic, clinical and laboratory features of the enrolled systemic lupus erythematosus patients, grouped according to disease activity identified by swollen to tender joint count ratio values.

\begin{tabular}{|c|c|c|c|c|}
\hline & \multicolumn{3}{|c|}{ STR } & \multirow[t]{2}{*}{$p$ value } \\
\hline & $\begin{array}{l}\text { Low } \\
\mathrm{N}=70\end{array}$ & $\begin{array}{l}\text { Moderate } \\
\mathrm{N}=23\end{array}$ & $\begin{array}{l}\text { High } \\
\mathrm{N}=7\end{array}$ & \\
\hline Male/Female & $3 / 67$ & $1 / 22$ & $1 / 6$ & \\
\hline Skin involvement, N (\%) & $41(58.6)$ & $16(59.2)$ & $6(85.7)$ & $\begin{array}{l}\text { H vs } M: p<0.0001 \\
H \text { vs L: } p<0.0001\end{array}$ \\
\hline NPSLE, N (\%) & $5(7.1)$ & $1(3.7)$ & $2(28.6)$ & $\begin{array}{l}\text { H vs } M: p<0.0001 \\
H \text { vs } L: p=0.0001\end{array}$ \\
\hline Renal involvement, N (\%) & $11(17.7)$ & $11(40.7)$ & $4(57.1)$ & $\begin{array}{l}\text { H vs } L: p<0.0001 \\
M \text { vs } L: p<0.0001\end{array}$ \\
\hline Anti-dsDNA, N (\%) & $40(57.1)$ & $14(51.8)$ & $6(85.7)$ & $\begin{array}{l}H \text { vs } L: p<0.0001 \\
H \text { vs } M: p<0.0001\end{array}$ \\
\hline Anti-SSA, N (\%) & $25(35.7)$ & $7(25.9)$ & $4(57.1)$ & $\begin{array}{l}H \text { vs } L: p=0.0002 \\
H \text { vs } M: p<0.0001\end{array}$ \\
\hline Anti-cardiolipin, N (\%) & $19(27.1)$ & $3(11.1)$ & 0 & L vs $\mathrm{M}: \mathrm{p}=0.006$ \\
\hline Low C3 complement, N (\%) & $26(37.1)$ & $14(51.8)$ & $4(57.1)$ & $H$ vs $L: p=0.006$ \\
\hline Low C4 complement, N (\%) & $17(24.3)$ & $10(37.0)$ & $3(42.8)$ & $H$ vs $L: p=0.006$ \\
\hline \multicolumn{5}{|l|}{ Previous treatments } \\
\hline MMF, N (\%) & $21(30.0)$ & $10(37.0)$ & $4(57.1)$ & $\begin{array}{l}\text { H vs } M: p=0.006 \\
H \text { vs } L: p=0.0001\end{array}$ \\
\hline \multicolumn{5}{|l|}{ Concomitant treatments } \\
\hline $\mathrm{HCQ}, \mathrm{N}(\%)$ & 45 (64.3) & $11(40.7)$ & $6(85.7)$ & $\begin{array}{l}\text { H vs } M: p<0.0001 \\
\text { H vs L: } p=0.001\end{array}$ \\
\hline
\end{tabular}

STR, swollen to tender joint count ratio; $\mathrm{H}$, high; $\mathrm{M}$, moderate; NPSLE, neuropsychiatric systemic lupus erythematosus; L, low; MMF, mycophenolate mofetil; $\mathrm{HCQ}$, hydroxychloroquine.

A

$100,0 \%$

$80,0 \%$

$60,0 \%$

$40,0 \%$

$20,0 \%$

$0,0 \%$
B

$100,0 \%$

$80,0 \%$

$60,0 \%$

$40,0 \%$

$20,0 \%$

$0,0 \%$

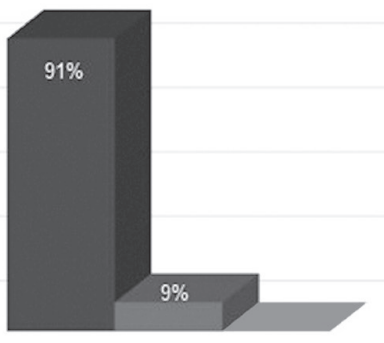

Figure 2 - Distribution of the three subset of disease activity according to swollen to tender joint count ratio values in patients with $(A)$ and without joint involvement defined by systemic lupus erythematosus disease activity index 2000 (B). 
erogeneous clinical features (8-11). The therapeutic strategy in SLE patients should include the control of disease activity and the prevention of chronic damage $(12,13)$. Joint involvement can affect up to $90 \%$ of SLE patients $(1,2)$. It is associated with different degrees of severity ranging from mild arthralgia to erosive disease (2). Data from the literature suggest a prevalent polyarticular involvement and inflammatory signs even in asymptomatic joints (14, $15)$.

Moreover, the assessment of disease activity and treatment response is crucial in the management of SLE patients with prevalent joint involvement. Several global indexes have been proposed and validated to assess disease activity in SLE patients. The revised SLEDAI-2K is the most frequently used in the clinical practice as well as in observational studies, due to its simplicity and feasibility (3). Among the SLEDAI items, joint involvement is defined as the presence of at least 2 joints with pain and signs of inflammation (i.e., tenderness, swelling or effusion), with a corresponding score of 4 (3). This value is not random in that it highlights the importance of joint involvement, because the disease is considered to be active with a SLEDAI-2K value $\geq 4$. Nonetheless, it is also clear that the definition in the SLEDAI-2K cannot capture all the potential features of joint involvement in SLE patients and cannot therefore reflect fully the evolution of joint involvement during the follow-up.

While evaluating the efficacy of methotrexate and chloroquine in the treatment of 41 SLE patients, Islam et al. observed a significant reduction of swollen and tender joint counts, morning stiffness, articular pain, and an improvement in the physician's and patient's global assessment after 24 weeks of treatment (16). Castrejon et al. applied the rheumatoid arthritis disease activity index (RADAI) in a cohort of patients affected by different rheumatic diseases other than RA. Fifty-nine percent of the 75 SLE patients included in the study reported the involvement of at least one joint (17).

More recently, we identified a moderate/ high level of disease activity using DAS28 in up to $60 \%$ of 69 SLE patients with joint involvement, and remission only in $11.6 \%$ (18). Moreover, $69.6 \%$ of the patients, classified without joint involvement according with the SLEDAI-2K, had an active disease on the basis of the DAS28 calculation (18). Unfortunately, the DAS28 depends on ESR and $\mathrm{GH}$, which could be influenced by other concomitant clinical features. Kristensen et al. found that the STR, which does not consider ESR or GH, was able to monitor successfully the response to the biological treatment in patients with RA (4). Interestingly, we found an association between STR and DAS28 values in our cohort of SLE patients with joint involvement. This finding appears very attractive because it suggests the possibility of using an index that can capture joint involvement without including parameters which can be potentially influenced by other disease-related factors, such as ESR and visual analogue scale.

We found a surprisingly high number $(9 \%)$ of patients without joint involvement according to SLEDAI-2K, who, however, scored a moderate STR. Therefore, also in the light of some results from previous studies (4), this small yet significant sub-population could potentially start a treatment for joint involvement, despite this symptom is not included in the SLEDAI-2K. Moreover, the degrees of activity identified by the STR seem to be associated with different disease manifestations. In particular, patients with a high STR showed more frequently neuropsychiatric and renal involvement compared to those with moderate and low activity. Also the positivity for anti-dsDNA and anti-SSA antibodies was significantly more frequent in SLE with high STR-disease activity.

It should be considered that the median STR score is low in this cohort and the majority of patients showed a low activity score, indicating a greater number of tender joints compared with swollen ones. We cannot exclude a possible role of a concomitant fibromyalgia, which was not assessed in this analysis.

In conclusion, our study suggests the possibility of using the STR in the assessment of 
joint involvement in SLE patients. One of the strengths of this index is that it can be easily applied in the clinical practice, thus allowing a quick assessment. At the same time, its sensitivity demonstrated in previous studies on RA patients (4) suggests the need for longitudinal studies in larger populations of SLE patients.

Competing interest: Nothing to declare.

\section{REFERENCES}

1. Grossman JM. Lupus arthritis. Best Pract Res Clin Rheumatol. 2009; 23: 495-506.

2. Santiago MB, Galvão V. Jaccoud arthropathy in systemic lupus erythematosus: analysis of clinical characteristics and review of the literature. Medicine (Baltimore). 2008; 87: 3744.

3. Gladman DD, Ibañez D, Urowitz MB. Systemic lupus erythematosus disease activity index 2000. J Rheumatol. 2002; 29: 288-91.

4. Kristensen LE, Bliddal H, Christensen R, Karlsson JA, Gülfe A, Saxne T, et al. Is swollen to tender joint count ratio a new and useful clinical marker for biologic drug response in rheumatoid arthritis? Results from a Swedish cohort. Arthritis Care Res (Hoboken). 2014; 66: 173-9.

5. Hochberg MC. Updating the American College of Rheumatology revised criteria for the classification of systemic lupus erythematosus. Arthritis Rheum. 1997; 40: 1725.

6. Prevoo ML, van 't Hof MA, Kuper HH, van Leeuwen MA, van de Putte LB, van Riel PL. Modified disease activity scores that include twenty-eight-joint counts. Development and validation in a prospective longitudinal study of patients with rheumatoid arthritis. Arthritis Rheum. 1995; 38: 44-8.

7. Fransen J, Creemers MC, Van Riel PL. Remission in rheumatoid arthritis: agreement of the disease activity score (DAS28) with the ARA preliminary remission criteria. Rheumatology (Oxford). 2004; 43: 1252-5.

8. Tsokos GC. Systemic lupus erythematosus. N Engl J Med. 2011; 365: 2110-21.

9. Ciccacci C, Perricone C, Ceccarelli F, Rufini S, Di Fusco D, Alessandri C, et al. A multilocus genetic study in a cohort of Italian SLE patients confirms the association with STAT4 gene and describes a new association with HCP5 gene. PLoS One. 2014; 9: e111991.

10. Sherer Y, Gorstein A, Fritzler MJ, Shoenfeld Y. Autoantibody explosion in systemic lupus erythematosus: more than 100 different antibodies found in SLE patients. Semin Arthritis Rheum. 2004; 34: 501-37.

11. Colasanti T, Maselli A, Conti F, Sanchez M, Alessandri C, Barbati C, et al. Autoantibodies to estrogen receptor $\alpha$ interfere with $\mathrm{T}$ lymphocyte homeostasis and are associated with disease activity in systemic lupus erythematosus. Arthritis Rheum. 2012; 64: 778-87.

12. Conti F, Ceccarelli F, Perricone C, Massaro L, Cipriano E, Pacucci VA, et al. Mycophenolate mofetil in systemic lupus erythematosus: results from a retrospective study in a large monocentric cohort and review of the literature. Immunol Res. 2014; 60: 270-6.

13. Conti F, Ceccarelli F, Massaro L, Cipriano E, Di Franco M, Alessandri C, et al. Biological therapies in rheumatic diseases. Clin Ter. 2013; 164: e413-28.

14. Iagnocco A, Ceccarelli F, Rizzo C, Truglia S, Massaro L, Spinelli FR, et al. Ultrasound evaluation of hand, wrist and foot joint synovitis in systemic lupus erythematosus. Rheumatology (Oxford). 2014; 53: 465-72.

15. Iagnocco A, Ossandon A, Coari G, Conti F, Priori R, Alessandri C, Valesini G. Wrist joint involvement in systemic lupus erythematosus. An ultrasonographic study. Clin Exp Rheumatol. 2004; 22: 621-4.

16. Islam MN, Hossain M, Haq SA, Alam MN, Ten Klooster PM, Rasker JJ. Efficacy and safety of methotrexate in articular and cutaneous manifestations of systemic lupus erythematosus. Int J Rheum Dis. 2012; 15: 62-8.

17. Castrejón I, Yazici Y, Pincus T. Patient selfreport RADAI (Rheumatoid Arthritis Disease Activity Index) joint counts on an MDHAQ (Multidimensional Health Assessment Questionnaire) in usual care of consecutive patients with rheumatic diseases other than rheumatoid arthritis. Arthritis Care Res (Hoboken). 2013; 65: 288-93.

18. Ceccarelli F, Perricone C, Massaro L, Pacucci VA, Cipriano E, Truglia S, et al. The role of disease activity score 28 in the evaluation of articular involvement in systemic lupus erythematosus. Sci World J. 2014; 2014: 236842. 